\title{
Astrocytic tumour grading: a comparative study of three-dimensional pseudocontinuous arterial spin labelling, dynamic susceptibility contrast-enhanced perfusion-weighted imaging, and diffusion-weighted imaging
}

\author{
Hua-Feng Xiao ${ }^{1} \cdot$ Zhi-Ye Chen $^{2} \cdot$ Xin Lou ${ }^{2,5} \cdot$ Yu-Lin Wang ${ }^{2} \cdot$ Qiu-Ping Gui ${ }^{3}$. \\ Yan Wang $^{2} \cdot$ Kai-Ning Shi $^{4} \cdot$ Zhen-Yu Zhou $^{4} \cdot$ Dan-Dan Zheng ${ }^{4} \cdot$ Danny J. J. Wang ${ }^{5}$. \\ Lin $\mathrm{Ma}^{2}$
}

Received: 27 January 2015 /Revised: 9 March 2015 / Accepted: 2 April 2015 / Published online: 23 May 2015

(C) The Author(s) 2015. This article is published with open access at Springerlink.com

\begin{abstract}
Objectives We hypothesized that three-dimensional pseudocontinuous arterial spin labelling (pCASL) may have similar efficacy in astrocytic tumour grading as dynamic susceptibility contrast-enhanced perfusion-weighted imaging (DSC-PWI), and the grading accuracy may be further improved when combined with apparent diffusion coefficient (ADC) values.

Methods Forty-three patients with astrocytic tumours were studied using diffusion weighted imaging (DWI), pCASL, and DSC-PWI. Histograms of ADC and normalized tumour cerebral blood flow values (nCBF on pCASL and $\mathrm{nrCBF}$ on DSC-PWI) were measured and analyzed.

Results The mean $10 \%$ ADC value was the DWI parameter that provided the best differentiation between low-grade astrocytoma (LGA) and high-grade astrocytoma (HGA). The $\mathrm{nCBF}$ and $\mathrm{nrCBF}(1.810 \pm 0.979$ and $2.070 \pm 1.048)$ in LGA were significantly lower than those $(4.505 \pm 2.270$ and $5.922 \pm$
\end{abstract}

Xin Lou

louxin301@gmail.com

Lin Ma

cjr.malin@vip.163.com

1 Department of Radiology, 302 Hospital of Chinese People's Liberation Army, Beijing 100039, China

2 Department of Radiology, People's Liberation Army General Hospital, No. 28 Fuxing Road, Beijing 100853, China

3 Department of Pathology, People's Liberation Army General Hospital, Beijing, China

4 General Electric Healthcare (China) Co., Ltd., Beijing, China

5 Department of Neurology, University of California, Los Angeles, CA, USA
2.630) in HGA. For differentiation between LGA and HGA, the cutoff values of $0.764 \times 10^{-3} \mathrm{~mm}^{2} / \mathrm{s}$ for mean $10 \% \mathrm{ADC}$, 2.374 for $\mathrm{nCBF}$, and 3.464 for $\mathrm{nrCBF}$ provided the optimal accuracy $(74.4 \%, 86.1 \%$, and $88.6 \%$, respectively). Combining the ADC values with nCBF or nrCBF could further improve the grading accuracy to $97.7 \%$ or $95.3 \%$, respectively.

Conclusions pCASL is an alternative to DSC-PWI for astrocytic tumour grading. The combination of DWI and contrastfree pCASL offers a valuable choice in patients with risk factors.

Key Points

- $p C A S L$ shows positive correlation with DSC-PWI in astrocytic tumour grading.

- ADC values based on ADC histograms can be an objective method.

- Combination of DWI and pCASL or DSC-PWI can improve grading accuracy.

Keywords Three-dimensional pseudocontinuous arterial spin labelling · Dynamic susceptibility contrast-enhanced perfusion-weighted imaging - Diffusion-weighted imaging . Astrocytic tumours $\cdot$ Grading
Abbreviations
HGA high-grade astrocytoma
LGA low-grade astrocytoma
nCBF normalized lesion/normal tissue $\mathrm{CBF}$
nrCBF normalized lesion/normal tissue $\mathrm{rCBF}$
pCASL pseudocontinuous arterial spin labelling
$\mathrm{rCBF}$ relative $\mathrm{CBF}$
ROC receiver operating characteristic 


\section{Introduction}

Astrocytic tumours of the central nervous system are classified by the World Health Organization into four grades according to pathological appearance. The tumour grading is of great importance in predicting the biological behaviour of the tumours and offers important guidance for accurate tumour biopsy and therapeutic decisions such as surgery, adjuvant radiation, and chemotherapy protocols [1], especially in non-resectable tumours. For these reasons, numerous efforts have been made to assess the astrocytic tumour grading using noninvasive imaging methods. Currently, conventional magnetic resonance imaging (MRI) techniques using T2-weighted image (T2WI), fluid attenuated inversion recovery (FLAIR), and pre-contrast and post-contrast T1-weighted image (T1WI) scans are routinely used for the examination of brain tumours, but accurate assessment of tumour grading cannot be achieved in some cases.

Some of the advanced MRI techniques, such as diffusion weighted imaging (DWI) and dynamic susceptibility contrastenhanced perfusion-weighted imaging (DSC-PWI), have shown promise in brain tumour grading [1-4]. By providing information derived from the microscopic motion of water molecules, the minimum apparent diffusion coefficient (ADC) values obtained by manually drawn regions of interest (ROIs) on an ADC map can enable the assessment of tumour cellularity and proliferation [4]. Accordingly, minimum ADC values can be used for brain tumour grading. DSC-PWI has been widely used as a valuable method for brain tumour grading, since it can be used to assess the tumour neovascularity. A previous DSC-PWI study suggested that the maximum relative cerebral blood volume $(\mathrm{rCBV})$ and relative cerebral blood flow (rCBF) correlated well with the brain tumour grades [5]. But the application of DSC-PWI involved the use of contrast media injection, which could cause complications, including nephrogenic systemic fibrosis in patients with chronic kidney disease. Arterial spin labelling (ASL) technique, however, is an entirely noninvasive alternative to DSC-PWI, and it could be categorized as either a continuous or pulsed ASL technique [6]. A newly developed ASL sequence, three-dimensional pseudocontinuous arterial spin labelling (pCASL), has potentially combined the advantages of pulsed ASL and continuous ASL, and has attracted significant attention [2, 7-9].

In the present study, $\mathrm{ADC}$ values were assessed using an $\mathrm{ADC}$ histogram to avoid the possible bias arising from the manual selection of ROIs from the ADC map. Meanwhile, cerebral blood flow (CBF) using pCASL, and rCBF using DSC-PWI were also investigated. The accuracy of brain tumour grading by each technique, as well as the combination of $\mathrm{ADC}$ values and contrast-free pCASL sequences, was investigated in a cohort of patients with astrocytic tumours.

\section{Materials and methods}

\section{Subjects}

This prospective study was approved by institutional ethics committee, and written informed consent was obtained from all the adult patients or from the parents of the paediatric patients. Between May 2012 and September 2014, 43 consecutive patients with suspected astrocytic tumours and preoperative MR imaging examinations including conventional MRI, DWI, pCASL, and DSC-PWI were included in the study. There were 25 males and 18 females, and their age ranged from 6 to 74 years, with a mean age of 43.3 years. All patients received preoperative assessment of internal carotid arteries using MR angiography and there were no significant stenoses. Laboratory tests showed normal renal function in all patients.

\section{MR imaging protocols}

All MR images were obtained on a 3.0 T MR scanner (Discovery 750, GE Healthcare, Milwaukee, WI, USA) with a 32channel head coil. Conventional MR scans included precontrast axial T1WI, axial, sagittal, and coronal T2WI, and post-contrast T1WI. T1WI was performed using T1-FLAIR (repetition time/echo time/inversion time $[\mathrm{TR} / \mathrm{TE} / \mathrm{TI}]=1$, $750 \mathrm{~ms} / 24 \mathrm{~ms} / 780 \mathrm{~ms}$, slice thickness $=5.0 \mathrm{~mm}$, slice gap= $1.5 \mathrm{~mm}$, field of view $=24 \times 18 \mathrm{~cm}$, matrix $=320 \times 320$, and NEX $=1.0$ ). Axial T2WI was performed using FRFSE (fast recovery fast spin echo) $(\mathrm{TR} / \mathrm{TE}=4,526 \mathrm{~ms} / 111 \mathrm{~ms}$, slice thickness $=5.0 \mathrm{~mm}$, slice gap $=1.5 \mathrm{~mm}$, field of view $=24 \times$ $24 \mathrm{~cm}$, matrix $=192 \times 192$, echo train length $=32$, and NEX $=$ 1.5). Sagittal and coronal T2WI were performed using T2FLAIR $(\mathrm{TR} / \mathrm{TE} / \mathrm{TI}=8,500 \mathrm{~ms} / 163 \mathrm{~ms} / 2,100 \mathrm{~ms}$, slice thickness $=5.0 \mathrm{~mm}$, slice gap $=1.5 \mathrm{~mm}$, field of view $=20 \times 20 \mathrm{~cm}$, matrix $=288 \times 224$, and NEX $=1.0$ ).

DWI was performed using fat-suppressed single-shot spinecho echo-planar imaging $(\mathrm{TR} / \mathrm{TE}=6,000 \mathrm{~ms} / 65.7 \mathrm{~ms}$, slice thickness $=5.0 \mathrm{~mm}$, slice gap $=1.5 \mathrm{~mm}$, field of view $=24 \times$ $24 \mathrm{~cm}$, matrix $=192 \times 192$, NEX $=2$ ) with $b=1,000 \mathrm{~s} / \mathrm{mm}^{2}$ applied in the $\mathrm{x}, \mathrm{y}$, and $\mathrm{z}$ directions, and $\mathrm{b}=0 \mathrm{~s} / \mathrm{mm}^{2}$ without motion-probing gradients, followed by automatic generation of isotropic DWI.

pCASL was performed using a background suppressed 3D fast spin echo (FSE) technique. The parameters were as follows: $\mathrm{TR}=4,653 \mathrm{~ms}$; $\mathrm{TE}=10.5 \mathrm{~ms}$; post-labelling delay $=1$, $525 \mathrm{~ms}$; FOV $=24 \times 24 \mathrm{~cm}$; matrix $=512 \times 512$; slice thickness $=4.0 \mathrm{~mm}$; slice gap $=0 \mathrm{~mm}$; NEX $=2$, and 36 pairs of tag and control images were acquired in $4 \min 30 \mathrm{~s}$. The imaging position extended from the vertex to the base of the skull.

DSC-PWI was then performed with single-shot gradientrecalled echo-planar imaging (GRE-EPI) sequence $(\mathrm{TR} / \mathrm{TE}=$ $1,525 \mathrm{~ms} /$ minimum, $250 \mathrm{~Hz} /$ pixel bandwidth for the 
acquisition of 20 axial sections, $\mathrm{FOV}=24 \times 24 \mathrm{~cm}, 1.0 \times 1.0 \times$ $5 \mathrm{~mm}$ voxel size, flip angle $=60^{\circ}$, matrix $=128 \times 128$, slice thickness $=5 \mathrm{~mm}$, slice gap $=1.5 \mathrm{~mm}, \mathrm{NEX}=1$, and number of slices $=20$ for 60 phases). Sixty images were obtained for each slice. Fat saturation was used to optimize the signal-tonoise ratio and baseline values. After five phases, $0.1 \mathrm{mmol} /$ kg Gd-DTPA (Bayer Schering Pharma AG, Berlin, Germany) was injected at a rate of $3 \mathrm{ml} / \mathrm{sec}$, immediately followed by a $20 \mathrm{ml}$ bolus of saline at the same injection rate. The total imaging time was $1 \mathrm{~min} 32 \mathrm{~s}$. Preload injection of contrast media before DSC-PWI acquisition was not applied.

Post-contrast T1WI was finally performed using FSPGR (fast spoiled gradient recalled echo) $(\mathrm{TR} / \mathrm{TE} / \mathrm{TI}=6.2 \mathrm{~ms} /$ $2.8 \mathrm{~ms} / 18 \mathrm{~ms}$, slice thickness $=4.0 \mathrm{~mm}$, slice gap $=0 \mathrm{~mm}$, field of view $=24 \times 24 \mathrm{~cm}$, matrix $=288 \times 224)$ in axial, sagittal, and coronal planes.

\section{MR data processing}

$\mathrm{ADC}, \mathrm{CBF}$ and $\mathrm{rCBF}$ color maps were post-processed using Functool software (GE Healthcare, Milwaukee, WI, USA) on a SUN GE AW 4.5 workstation. ADC, CBF, and rCBF measurements were conducted by a neuroradiologist with 5 years' experience in neuroradiology. Patient-related information was removed from all images, and the observer was blinded to the pathological diagnosis. All values were measured again with an interval of 3 months, and the intraobserver agreement was evaluated. The average values of initial and second measurements were regarded as the final $\mathrm{ADC}$, normalized $\mathrm{CBF}$ (nCBF), and normalized $\mathrm{rCBF}(\mathrm{nrCBF})$ values.

In the present study, an ADC histogram was generated and the parameters involving the entire lesion were used for acquisition. Volume rendering was used to retrieve the ADC map. The tumour volume was obtained in each slice of the axial, coronal, and sagittal planes by identifying the tumour contour with a colored brush and using the histogram pattern to obtain the tumour's ADC histogram. The tumour margins were defined by using conventional T2WI (FRFSE and FLAI $\mathrm{R}$ ) and post-contrast T1WI, and the necrotic/cystic or hemorrhagic regions in the tumours defined by conventional T2WI, pre-contrast and post-contrast T1WI were avoided in ADC images. By manual placing the metering rod at the lower $10 \%, 25 \%$, and $50 \%$ on ADC histogram curve, the mean $10 \%$, mean $25 \%$, mean $50 \%$, mean, minimum, and maximum ACD values were determined (Figs. 1, 2).

Maximal perfusion values, including maximal CBF (pCASL) and maximal rCBF (DSC-PWI) of the tumours, were measured as follows: on all the continuous pCASL (Figs. 1e, 2e) and DSC-PWI slices (Figs. 1g, 2g), five to eight round ROIs $\left(28-32 \mathrm{~mm}^{2}\right)$ were manually and carefully drawn in the deep color areas within the solid tumour portions on each slice, and the ROIs with the highest $\mathrm{CBF}$ and $\mathrm{rCBF}$ values were selected from the whole tumour. Those $\mathrm{CBF}$

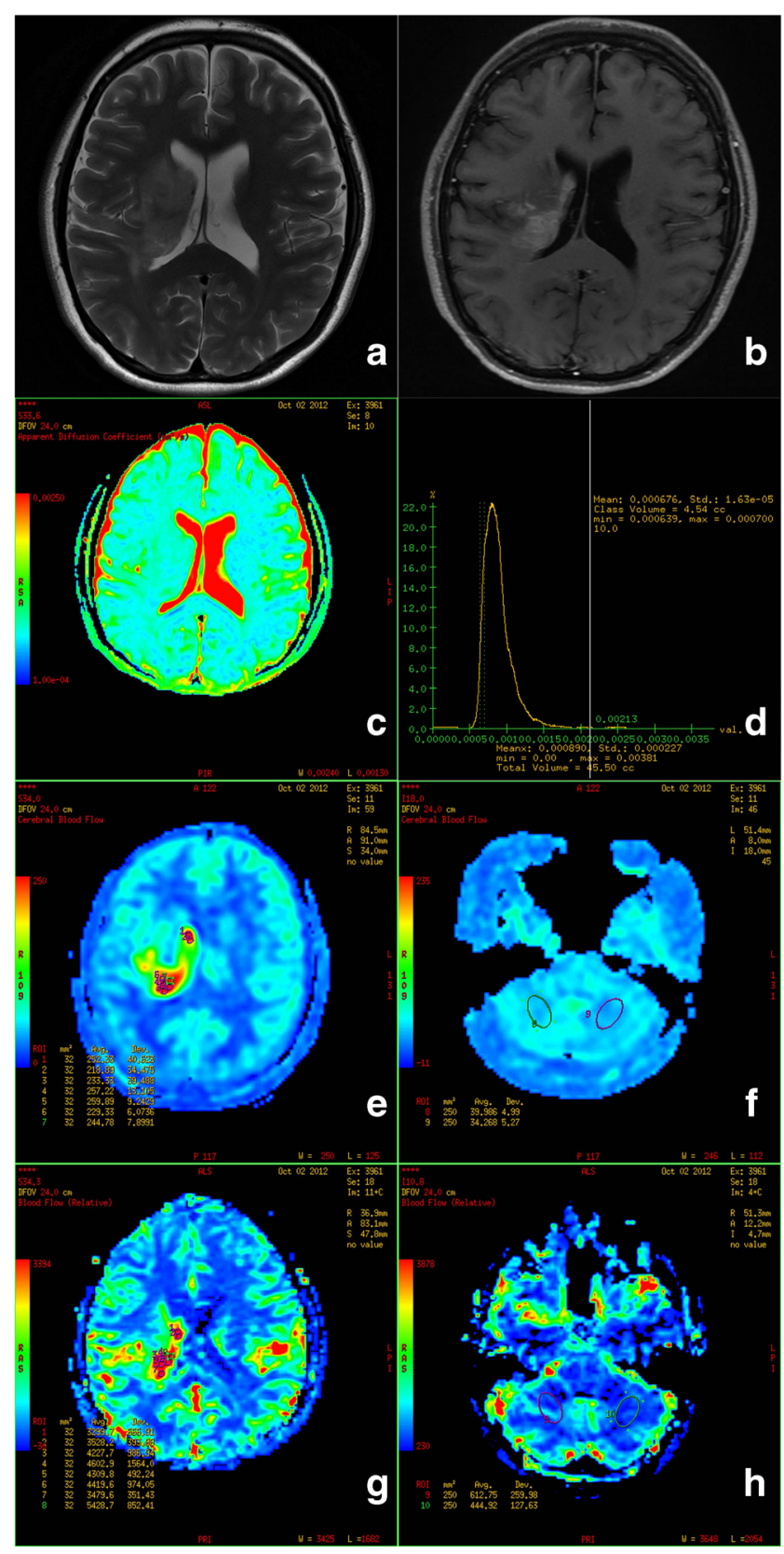

Fig. 1 A sixteen-year-old girl with anaplastic astrocytoma (WHO III) in the right basal ganglion and thalamus. $\boldsymbol{a}$ The tumour showed hyperintense signal on T2WI. $\boldsymbol{b}$ The tumour showed patchy enhancement on postcontrast T1WI. $\boldsymbol{c}$ ADC map. $\boldsymbol{d}$ ADC histogram of the tumour. $\boldsymbol{e}$ ROI selection for the maximal $\mathrm{CBF}$ of the tumour on pCASL CBF color map. $\boldsymbol{f}$ ROI selection for bilateral cerebellum CBF on pCASL CBF color map. $g$ ROI selection for the maximal $\mathrm{rCBF}$ of the tumour on DSC-PWI rCBF color map. $\boldsymbol{h}$ ROI selection for bilateral cerebellum rCBF on DSC-PWI rCBF color map

and $\mathrm{rCBF}$ values were regarded as the final maximal values. Since some DSC-PWI and PET studies have verified that cerebellar perfusion is usually stable and unaffected by pathology $[8,10,11]$, the normalization with the cerebellum was used in the present study, and nCBF on pCASL and nrCBF on DSCPWI were defined as the mean value for ROI in a tumour area 


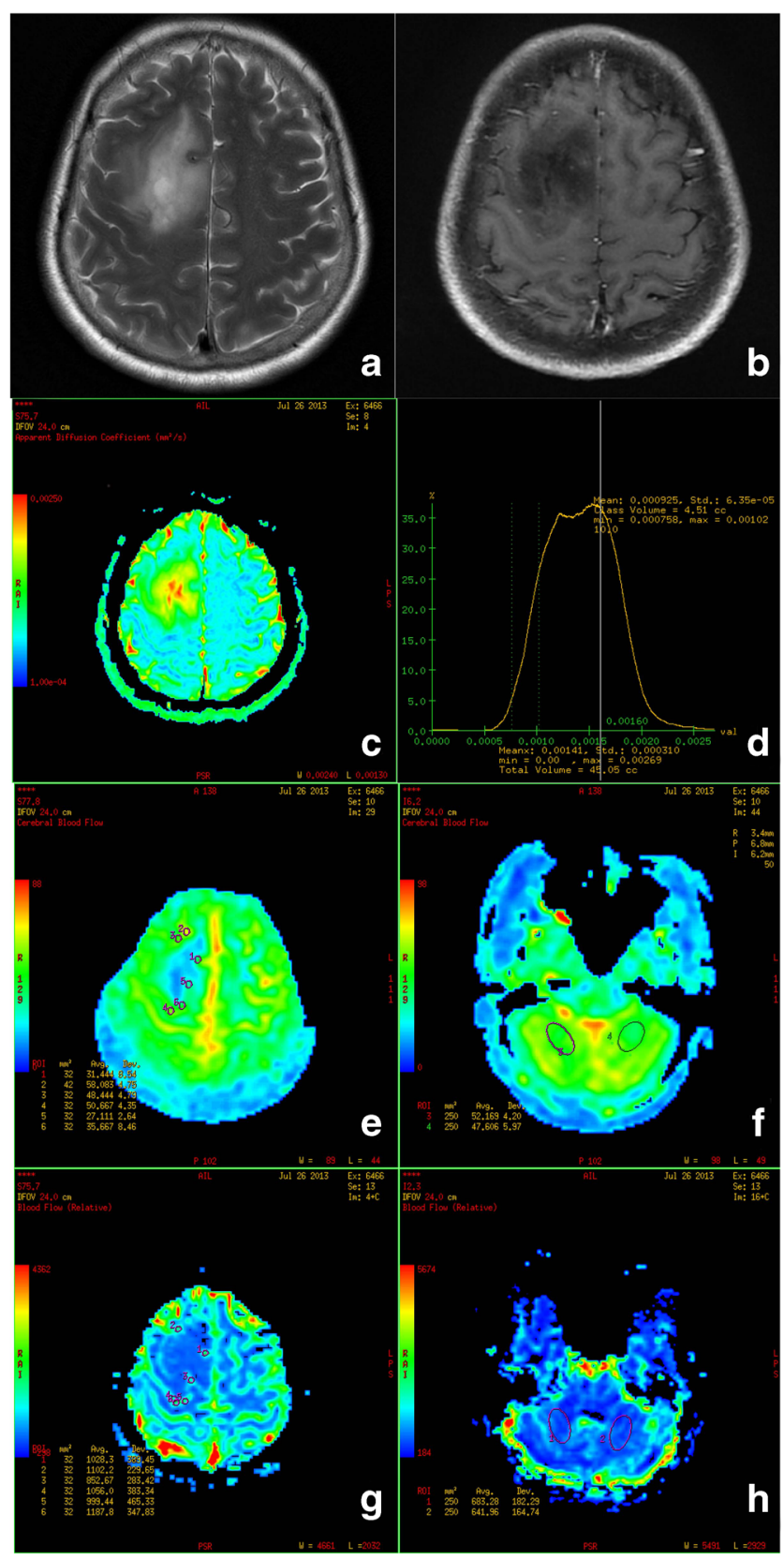

Fig. 2 A sixty-year-old woman with diffuse astrocytoma (WHO II) in the right frontal lobe. $\boldsymbol{a}$ The tumour showed hyperintense signal on T2WI. $\boldsymbol{b}$ The tumour demonstrated no enhancement on post-contrast T1WI. $c$ ADC map. $\boldsymbol{d}$ ADC histogram of the tumour. $\boldsymbol{e}$ ROI selection for the maximal $\mathrm{CBF}$ of the tumour on pCASL CBF color map. $f$ ROI selection for bilateral cerebellum $\mathrm{CBF}$ on pCASL CBF color map. $g$ ROI selection for the maximal rCBF of the tumour on DSC-PWI rCBF color map. $\boldsymbol{h}$ ROI selection for bilateral cerebellum rCBF on DSC-PWI rCBF color map

with maximal perfusion/mean value for ROI in cerebellum. Cerebellar ROIs (approximately $250 \mathrm{~mm}^{2}$ on each side) were placed in the central regions of the bilateral cerebellar hemispheres (Figs. 1-2f, h), and the mean value was regarded as the reference. Cerebellar reference ROIs were not influenced by cerebellar tumours (one pilocytic astrocytoma) in this study because of the small tumour size.

\section{Pathological observation}

All patients underwent navigation-guided $(\mathrm{n}=28)$ or open surgery $(n=15)$ within 7 days after the MR scan. For both surgical approaches, the tumour portions with maximal perfusion on MR imaging were used for guidance and should be included in the surgical resection. All specimens obtained for pathological observation were fixed in formalin and embedded in paraffin. Hematoxylin and eosin-stained and immunohistochemically prepared specimens were performed to determine the histological type of the tumour after surgery. The pathological diagnosis was made by a pathologist with 20 years of experience in neuropathology. According to the World Health Organization (WHO) criteria [12], tumours of WHO grade I and II were classified as low-grade astrocytoma (LGA), whereas tumours of WHO grade III and IV were classified as high-grade astrocytoma (HGA).

\section{Statistical analysis}

Intraobserver agreement was assessed with the intraclass correlation coefficient (ICC), in which ICC $<0.40=$ poor, 0.40 $0.59=$ fair, $0.60-0.74=$ good, and $>0.74=$ excellent.

All statistical analyses were performed using the statistical package SPSS for Windows (Version 16.0, Chicago, IL, USA). The level of significance was set at $p<0.05$. ADC values, $\mathrm{nCBF}$ and $\mathrm{nrCBF}$ between LGA and HGA were analyzed and compared using one-way ANOVA and Student's unpaired t-test.

Pearson's correlation analysis was performed between $\mathrm{nCBF}$ and $\mathrm{nrCBF}$, between $\mathrm{ADC}$ values and $\mathrm{nCBF}$, and between $\mathrm{ADC}$ values and $\mathrm{nrCBF}$, respectively.

Receiver operating characteristic (ROC) curves for ADC values, $\mathrm{nCBF}$, and $\mathrm{nrCBF}$ were applied to assess the cutoff value that best balanced sensitivity and specificity to allow differentiation between LGA and HGA. Binary logistic regression was performed using mean $10 \% \mathrm{ADC}+\mathrm{nCBF}$, and mean $10 \% \mathrm{ADC}+\mathrm{nrCBF}$, respectively. In each patient, five predicted probabilities produced from mean $10 \% \mathrm{ADC}$, $\mathrm{nCBF}$, nrCBF, mean $10 \% \mathrm{ADC}+\mathrm{nCBF}$, and mean $10 \%$ $\mathrm{ADC}+\mathrm{nrCBF}$ were set as the variables, and each predicted probability was evaluated using ROC curve [13].

\section{Results}

\section{Pathological results}

Nineteen LGAs (one pilocytic astrocytoma and 18 diffuse astrocytomas) and 24 HGAs (six anaplastic astrocytomas and 18 glioblastomas) were proved by pathology. 


\section{ICC evaluation}

The intraobserver reproducibility of mean $10 \%$ ADC measurements on ADC histogram, nCBF measurements on 3DpCASL, and nrCBF measurements on DSC-PWI was excellent. ICCs were 0.927 for ADC values, 0.869 for $\mathrm{nCBF}$, and 0.850 for $\mathrm{nrCBF}$, respectively.

\section{ADC histogram parameters, $\mathrm{nCBF}$, and $\mathrm{nrCBF}$}

The ADC values of LGA were significantly higher than those of HGA using the mean $10 \%$, mean $25 \%$, mean, and minimum ADC values, and the difference was most significant by using the mean $10 \%$ ADC value $(p<0.001)$. The differences were not significant using the mean $50 \%$ and maximum ADC values (Table 1).

The $\mathrm{nCBF}(1.810 \pm 0.979)$ in the LGA group was significantly lower than that $(4.505 \pm 2.270)$ in the HGA group $(p<0.001)$, and the nrCBF $(2.070 \pm 1.048)$ in the LGA group was significantly lower than that $(5.922 \pm 2.630)$ in the HGA group $(p<0.001)$ (Table 2$)$.

Pearson's correlation analysis showed a significant positive correlation between $\mathrm{nCBF}$ and $\operatorname{nrCBF}(\mathrm{r}=0.751, p<0.001)$ (Fig. 3a). The mean $10 \%$ ADC value was chosen, and Pearson's correlation analysis showed a negative correlation between the mean $10 \% \mathrm{ADC}$ and $\mathrm{nCBF}(\mathrm{r}=-0.323, p<0.01)$ (Fig. 3b) and between mean $10 \%$ ADC and $\operatorname{nrCBF}(\mathrm{r}=-0.430$, $p<0.01$ ) (Fig. 3c).

\section{ROC analysis}

The results of the ROC analysis using the mean $10 \%$ ADC value, $\mathrm{nCBF}$, nrCBF, mean $10 \% \mathrm{ADC}+\mathrm{nCBF}$, and mean $10 \% \mathrm{ADC}+$ nrCBF for the differentiation between LGA and HGA were presented in Table 3 and Fig. 4. The accuracy was $74.4 \%$ for mean $10 \%$ ADC value, $86.1 \%$ for $\mathrm{nCBF}$, and $88.6 \%$ for nrCBF, respectively. Combining the cutoff value of mean $10 \%$ ADC with $\mathrm{nCBF}$ or nrCBF could improve the grading accuracy up to $97.7 \%$ or $95.3 \%$, respectively.

Table 1 ADC histogram values (mean \pm SD) $\left(\times 10^{-3} \mathrm{~mm}^{2} / \mathrm{s}\right)$ of LGA and HGA

\begin{tabular}{llll}
\hline & LGA $(n=19)$ & HGA $(n=24)$ & $p$ value \\
\hline Mean 10 \% ADC & $0.886 \pm 0.118$ & $0.745 \pm 0.079$ & $<0.001$ \\
Mean 25 \% ADC & $1.005 \pm 0.195$ & $0.844 \pm 0.089$ & 0.003 \\
Mean 50 \% ADC & $1.167 \pm 0.302$ & $1.239 \pm 0.108$ & 0.752 \\
Mean ADC & $1.418 \pm 0.375$ & $1.204 \pm 0.188$ & 0.019 \\
Minimum ADC & $0.625 \pm 0.071$ & $0.548 \pm 0.093$ & 0.038 \\
Maximum ADC & $3.437 \pm 0.578$ & $3.310 \pm 0.676$ & 0.519 \\
\hline
\end{tabular}

$\mathrm{SD}=$ standard deviation
Table 2 The $\mathrm{nCBF}$ and $\mathrm{nrCBF}($ mean $\pm \mathrm{SD}$ ) of LGAs and HGAs

\begin{tabular}{llll}
\hline & LGA $(\mathrm{n}=19)$ & HGA $(\mathrm{n}=24)$ & $p$ value \\
\hline $\mathrm{nCBF}$ & $1.810 \pm 0.979$ & $4.505 \pm 2.270$ & $<0.001$ \\
$\mathrm{nrCBF}$ & $2.070 \pm 1.048$ & $5.922 \pm 2.630$ & $<0.001$ \\
\hline
\end{tabular}

$\mathrm{SD}=$ standard deviation

According to the linear interpolation method, the diagnostic point based on the ROC was calculated as predicted probability for mean $10 \% \mathrm{ADC}+\mathrm{nCBF}(P 1)$ or predicted probability for mean $10 \% \mathrm{ADC}+\operatorname{nrCBF}(P 2)$. The best diagnostic points were 0.354 for $P 1$ and 0.488 for $P 2$, respectively, which meant if the predicted probability was higher than or equal to 0.354 or 0.488 , the tumour could be diagnosed as HGA rather than LGA, and vice versa. The logistic regression equation was built and is described as follows:

$$
\begin{aligned}
& P 1=\frac{1}{\left.1+\mathrm{e}^{-(15.544-2.657 \mathrm{E} 4 \mathrm{X} 1}+2.445 \mathrm{X} 2\right)} \\
& P 2=\frac{1}{\left.1+e^{-(19.758-3.027 \mathrm{E} 4 \mathrm{X} 1}+1.371 \mathrm{X} 3\right)}
\end{aligned}
$$

$\left(\mathrm{X}_{1}\right.$ : mean $10 \% \mathrm{ADC}$ value; $\mathrm{X}_{2}$ : $\mathrm{nCBF}$ value; $\mathrm{X}_{3}: \mathrm{nrCBF}$ value; E4: $10^{4}$ )

\section{Discussion}

In this study, we noted that a close correlation was presented between nCBF (3D-pCASL) and nrCBF (DSC-PWI) in astrocytic tumour grading, which was in agreement with the results of previous studies [14]. Although the overlap in nCBF or nrCBF at the cutoff values was noticed, most patients in the LGA group had values below the threshold, while most patients in the HGA group had values above the threshold. One grade II astrocytoma, which abutted the cerebral convexity and falx, showed obvious hyperperfusion on 3D-pCASL with an increased $\mathrm{nCBF}$ value of 4.88. The possible explanation could be that the tumour was closely related with the adjacent meningeal vessels. However, its nrCBF value of 1.58 was not as high as expected. Susceptibility of DSC-PWI may result in decreased perfusion in such superficial lesions.

A histogram is a plot in which widths represent class intervals and areas are proportional to the corresponding frequencies. The key point was to ensure that the tumour boundary should be outlined accurately by encompassing the entire tumour, avoiding necrosis, haemorrhage, and peripheral oedema. Although multiple parameters from the ADC histogram can be used to grade astrocytic tumours, the statistical results indicated that in this study the mean $10 \%$ ADC value was most significant in differentiating LGA from HGA. The cutoff value of mean $10 \% \mathrm{ADC}\left(0.764 \times 10^{-3} \mathrm{~mm}^{2} / \mathrm{s}\right)$ in this study was 

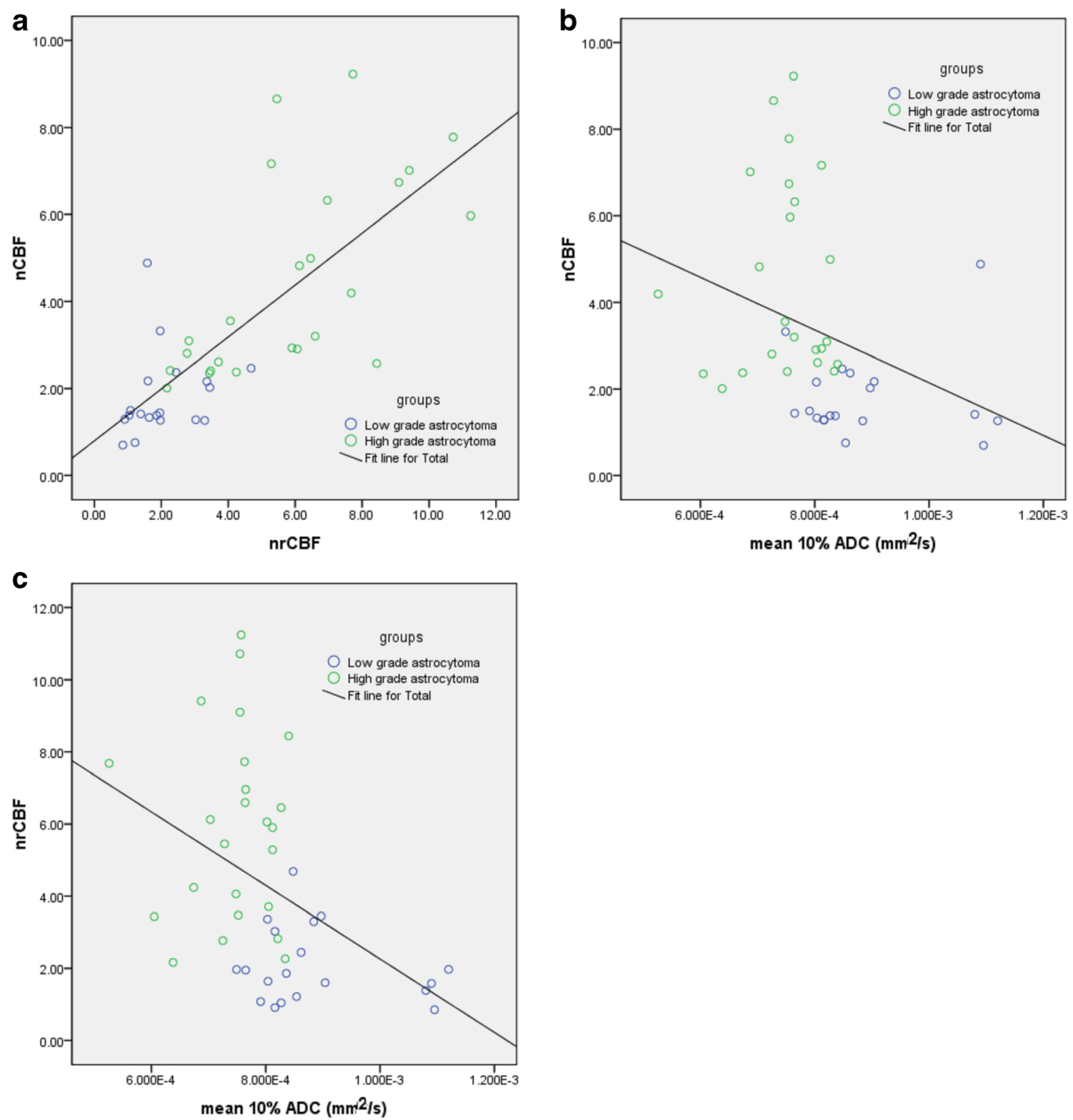

Fig. $3 \boldsymbol{a}$ Scatterplot of the correlation between the nCBF and nrCBF in LGAs and HGAs. $\boldsymbol{b}$ Scatterplot of the correlation between the mean $10 \%$ ADC value and nCBF in LGAs and HGAs. $c$ Scatterplot of the correlation between the mean $10 \%$ ADC value and the nrCBF in LGAs and HGAs

lower than those $\left(0.900 \times 10^{-3} \mathrm{~mm}^{2} / \mathrm{s}\right.$ and $\left.1.01 \times 10^{-3} \mathrm{~mm}^{2} / \mathrm{s}\right)$ reported in other studies based on manual ROI measurement
$[4,15]$. The discrepancy might be mainly due to the differences in the sampling methodology. Moreover, disruptions in the

Table 3 ROC analysis of the mean $10 \% \mathrm{ADC}\left(\times 10^{-3} \mathrm{~mm} 2 / \mathrm{s}\right), \mathrm{nCBF}, \mathrm{nrCBF}$, mean $10 \% \mathrm{ADC}+\mathrm{nCBF}$, and mean $10 \% \mathrm{ADC}+\mathrm{nrCBF}$ values for astrocytic tumour grading

\begin{tabular}{llllrr}
\hline & Cutoff value & AUC & Sensitivity & Specificity & Accuracy \\
\hline Mean $10 \%$ ADC & 0.764 & 0.875 & $94.7 \%$ & $62.5 \%$ & $74.4 \%$ \\
nCBF & 2.374 & 0.919 & $91.7 \%$ & $84.2 \%$ & $96.1 \%$ \\
nrCBF & 3.464 & 0.939 & $79.2 \%$ & $84.7 \%$ & N/A \\
Mean $10 \%$ ADC + nCBF & N/A & 0.974 & N/A & N/A & $97.7 \%$ \\
Mean $10 \%$ ADC + nrCBF & N/A & 0.974 & N/A & $95.3 \%$ \\
\hline
\end{tabular}

$\mathrm{N} / \mathrm{A}=$ not available 
ROC Curve

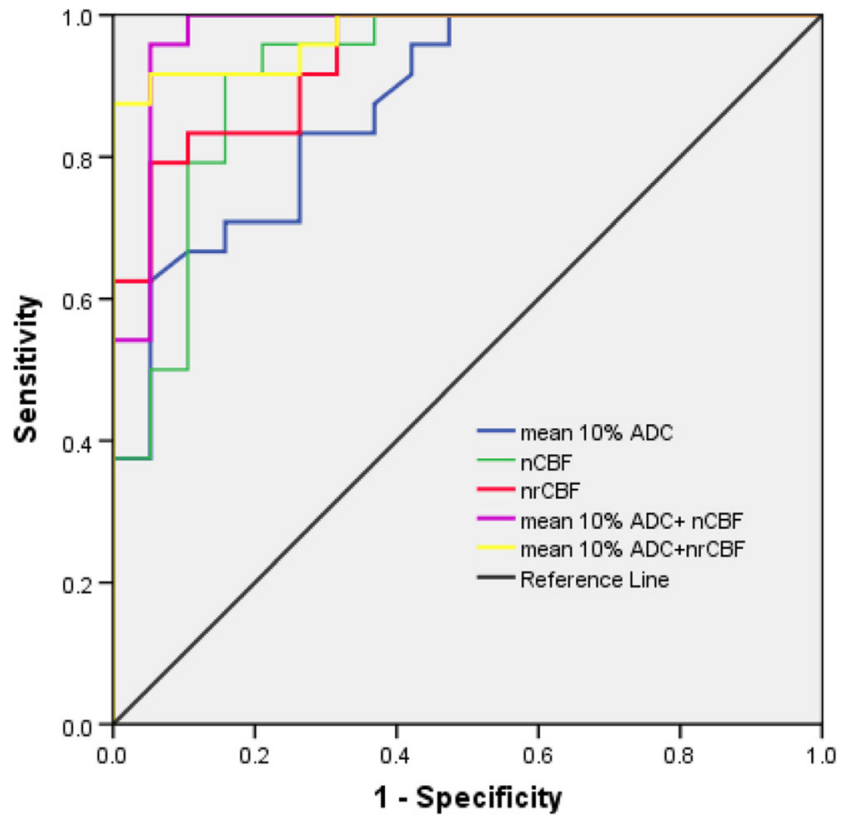

Fig. 4 ROC map of the mean $10 \%$ ADC, nCBF, nrCBF, mean $10 \%$ $\mathrm{ADC}+\mathrm{nCBF}$, and mean $10 \% \mathrm{ADC}+\mathrm{nrCBF}$ values for the discrimination of LGAs vs. HGAs

class intervals on histogram were found in some tumours, which could be compensated by using the mean $10 \%$ ADC value. Future study is needed to compare the accuracy between histogram-generated ADC values and manual ROI-defined ADC values in tumour grading $[4,15,16]$.

The present investigation demonstrated that an inverse correlation existed between mean $10 \% \mathrm{ADC}$ value and both $\mathrm{nCBF}$ and $\mathrm{nrCBF}$, suggesting that increased cellularity (low $\mathrm{ADC}$ ) was correlated with high vascularity (high $\mathrm{nCBF}$ or nrCBF) in astrocytic tumours. ADC value could be used in astrocytic tumour grading, which has been shown to reflect the histologically determined cellular density $[17,18]$. We believe that mean $10 \% \mathrm{ADC}$ value had intrinsic advantages, although its overall efficacy was inferior to pCASL and DSCPWI in this study. For example, the ADC value could be used to compensate for the decreased grading accuracy in some LGAs with high CBF or HGAs with low CBF. One aforementioned case of grade II astrocytoma with hyperperfusion on nCBF could be properly graded as LGA by using the mean $10 \%$ ADC value $\left(0.848 \times 10^{-3} \mathrm{~mm}^{2} / \mathrm{s}\right)$, which was higher than the cutoff value $\left(0.764 \times 10^{-3} \mathrm{~mm}^{2} / \mathrm{s}\right)[19]$. In this case, a correct tumour grading could also be achieved by using $P 2$ value (0.023), which was lower than the diagnostic point (0.488).

In this study, the accuracy for astrocytic tumour grading was improved by combining the mean $10 \%$ ADC value with perfusion-base techniques such as 3D-pCASL or DSC-PWI as compared with individual methods. Our results also indicated that the accuracy of astrocytic tumour grading by combined ADC value and perfusion parameters was higher than those in previous studies $[1,10,15,20]$. The authors considered that the use of predicted probabilities ( $\mathrm{P} 1$ and $\mathrm{P} 2$ values) was practicable in clinical practice. Moreover, when the patients were under the conditions of chronic kidney disease, dialysis, hyperphosphatemia, acidosis, and pro-inflammatory events, gadolinium-based contrast agents should be used with caution [21], and the combination of MR techniques, including ADC values and contrast-free pCASL, should be the preferred method of choice for tumour grading.

Limitations of the present study were as follows. 1) The sample size was not large enough to make a further discussion on histological subtypes of LGAs and HGAs. 2) Only one post-labelling delay time $(1,525 \mathrm{~ms})$ in pCASL was used, which may have potential influences on the evaluation of cerebral flow in some cases. 3) Uncorrected maps in pCASL were used. To decrease the partial volume effects of pCASL acquisitions, a previous study acquired both uncorrected maps and maps corrected for partial volume effects, which yielded similar results, and CBF maps corrected for partial volume effects were not feasible for clinical use [22]. 4) Different slice thickness existed between 3D pCASL and 2D DSC-PWI; therefore, voxel-wise correlation could not be achieved between them, but anatomical correlation could be determined in assessing the perfusion values. 5) A major concern about the calculation errors of nrCBF values with DSC-PWI is the leakage of contrast agent from the vasculature to the tumour. Attempts to correct the calculation errors include preload injection of a small amount of contrast agent, use of dual-echo pulse sequence and baseline subtraction, but none of them are considered to be the satisfactory method of choice. Therefore, DSC-PWI without preload of contrast agent are clinically acceptable.

In conclusion, the mean $10 \% \mathrm{ADC}$ value based on ADC histogram had its intrinsic advantages and could be an objective method in predicting astrocytic tumour grading. pCASL could be reliably applied in tumour grading, since it had a significant positive correlation with DSC-PWI. The combination of ADC values and pCASL or DSC-PWI could markedly improve the accuracy for astrocytic tumour grading. Particularly, the combination of ADC values and contrast-free pCASL sequences could offer a clinically valuable method of choice in patients with major risk factors and in patients who need follow-up studies.

Acknowledgements The scientific guarantor of this publication is Lin $\mathrm{Ma}$. The authors of this manuscript declare relationships with the following companies: GE Healthcare. The authors of this manuscript declare no relationships with any companies, whose products or services may be related to the subject matter of the article. The authors state that this work has not received any funding.

Zhi-Ye Chen kindly provided statistical advice for this manuscript. Institutional Review Board approval was obtained. Written informed consent was obtained from all subjects (patients) in this study. Methodology: prospective, diagnostic or prognostic study, performed at one institution. 
Open Access This article is distributed under the terms of the Creative Commons Attribution-NonCommercial 4.0 International License (http:// creativecommons.org/licenses/by-nc/4.0/), which permits any noncommercial use, distribution, and reproduction in any medium, provided you give appropriate credit to the original author(s) and the source, provide a link to the Creative Commons license, and indicate if changes were made.

\section{References}

1. Law M, Yang S, Wang H et al (2003) Glioma grading: sensitivity, specificity, and predictive values of perfusion MR imaging and proton MR spectroscopic imaging compared with conventional MR imaging. AJNR Am J Neuroradiol 24:1989-1998

2. Roy B, Awasthi R, Bindal A et al (2013) Comparative evaluation of 3-dimensional pseudocontinuous arterial spin labeling with dynamic contrast-enhanced perfusion magnetic resonance imaging in grading of human glioma. J Comput Assist Tomogr 37:321-326

3. Romano A, Rossi Espagnet MC, Calabria LF et al (2012) Clinical applications of dynamic susceptibility contrast perfusion-weighted MR imaging in brain tumours. Radiol Med 117:445-460

4. Chen Z, Ma L, Lou X et al (2010) Diagnostic value of minimum apparent diffusion coefficient values in prediction of neuroepithelial tumor grading. J Magn Reson Imaging 31:1331-1338

5. Barrett T, Brechbiel M, Bernardo M et al (2007) MRI of tumor angiogenesis. J Magn Reson Imaging 26:235-249

6. Petcharunpaisan S, Ramalho J, Castillo M (2010) Arterial spin labeling in neuroimaging. World J Radiol 2:384-398

7. Nielsen JF, Hernandez-Garcia L (2013) Functional perfusion imaging using pseudocontinuous arterial spin labeling with low-flipangle segmented 3D spiral readouts. Magn Reson Med 69:382-390

8. Jarnum H, Steffensen EG, Knutsson L et al (2010) Perfusion MRI of brain tumours: a comparative study of pseudo-continuous arterial spin labelling and dynamic susceptibility contrast imaging. Neuroradiology 52:307-317

9. Wheaton AJ, Miyazaki M (2012) Non-contrast enhanced MR angiography: physical principles. J Magn Reson Imaging 36:286-304

10. Thomsen H, Steffensen E, Larsson EM (2012) Perfusion MRI (dynamic susceptibility contrast imaging) with different measurement approaches for the evaluation of blood flow and blood volume in human gliomas. Acta Radiol 53:95-101
11. Arbab AS, Aoki S, Toyama K et al (2001) Brain perfusion measured by flow-sensitive alternating inversion recovery (FAIR) and dynamic susceptibility contrast-enhanced magnetic resonance imaging: comparison with nuclear medicine technique. Eur Radiol 11: 635-641

12. Fuller GN, Scheithauer BW (2007) The 2007 Revised World Health Organization (WHO) Classification of Tumours of the Central Nervous System: newly codified entities. Brain Pathol 17: 304-307

13. Chen Z, Feng F, Yang Y et al (2012) MR imaging findings of the corpus callosum region in the differentiation between multiple sclerosis and neuromyelitis optica. Eur J Radiol 81:3491-3495

14. Lehmann P, Monet $\mathrm{P}$, de Marco G et al (2010) A comparative study of perfusion measurement in brain tumours at 3 Tesla MR: arterial spin labeling versus dynamic susceptibility contrast-enhanced MRI. Eur Neurol 64:21-26

15. Murakami R, Hirai T, Sugahara T et al (2009) Grading astrocytic tumors by using apparent diffusion coefficient parameters: superiority of a one- versus two-parameter pilot method. Radiology 251: 838-845

16. Bilgili Y, Unal B (2004) Effect of region of interest on interobserver variance in apparent diffusion coefficient measures. AJNR Am J Neuroradiol 25:108-111

17. Gupta RK, Cloughesy TF, Sinha U et al (2000) Relationships between choline magnetic resonance spectroscopy, apparent diffusion coefficient and quantitative histopathology in human glioma. J Neurooncol 50:215-226

18. Rollin N, Guyotat J, Streichenberger N et al (2006) Clinical relevance of diffusion and perfusion magnetic resonance imaging in assessing intra-axial brain tumors. Neuroradiology 48:150-159

19. Rumboldt Z, Camacho DL, Lake D et al (2006) Apparent diffusion coefficients for differentiation of cerebellar tumors in children. AJNR Am J Neuroradiol 27:1362-1369

20. Kim MJ, Kim HS et al (2008) Diagnostic accuracy and interobserver variability of pulsed arterial spin labeling for glioma grading. Acta Radiol 49:450-457

21. Zou Z, Ma L, Li H (2009) Incidence of nephrogenic systemic fibrosis at Chinese PLA General Hospital. J Magn Reson Imaging 30:1309-1312

22. Binnewijzend MA, Kuijer JP, Benedictus MR et al (2013) Cerebral blood flow measured with 3D pseudocontinuous arterial spinlabeling MR imaging in Alzheimer disease and mild cognitive impairment: a marker for disease severity. Radiology 267:221-230 